\title{
Vision/Odometer Autonomous Navigation Based on RatSLAM for Land Vehicles
}

\author{
Xiaofeng He, Lilian Zhang, Junxiang Lian, Xiaoping Hu \\ College of Mechatronics and Automation, National University of Defense Technology, Changsha, \\ 410073, China
}

Keywords: autonomous navigation, RatSLAM, computer vision, odometer.

\begin{abstract}
This paper discusses the design of computer vision and odometer integrated autonomous navigation algorithm based on RatSLAM system for land vehicles. The research aims at testing the effectiveness of a vision/odometer bio-inspired navigation approach for land vehicle applications. Images and odometer measurements are inputted into the RatSLAM system. Odometer data can be used to improve the robustness of RatSLAM system significantly. The proposed system has been verified on real vision/odometer data collected in land vehicle tests. Campus round road experiments illustrate that the algorithm is effective and robust.
\end{abstract}

\section{Introduction}

Since GPS (Global Positioning System) and other GNSS(Global Navigation Satellites System) technologies have been applied more and more widely, it seems that vehicles cannot navigate without GNSS receivers. In fact, the shortcomings of GNSS are fateful and serious. GNSS signal is deficient when facing obstruction and interference. So GNSS is hard to use in some situations such as basement, "city valley" and woods. Therefore in nature, many animals possess magic navigation abilities without using GNSS. This is a classic kind of autonomous navigation system, being called as biological navigation system.

There is a famous approach to solve the navigation problem of robots. It is simultaneous localization and mapping (SLAM) technique. Many conventional probabilistic techniques are present to implement SLAM of robots, especially intelligent land vehicles. Kalman filtering (KF) is one of the most popular methods used for the process of information fusion. Unfortunately, it is difficult for KF to obtain the perfect precision of navigation systems because it needs exact noise models of processing and sensory. In fact, the noise models of navigation systems can't enough inerrant. Hence, researchers try to find other ways to solve the SLAM problem.

Michael John Milford proposed a kind of bio-inspired SLAM method, known as RatSLAM, which is based on biological navigation theories in the rodent hippocampus [1]. The system equipped with a single camera only was demonstrated to be useful and excellent in their road experiments.

Odometer is easy and cheap to be adopted to navigate land vehicles. This paper discusses the design of a RatSLAM system not only with vision information, but also with odometer data which provides the velocity of land vehicles. Some outdoor experimental results show that the proposed algorithm is feasible.

\section{RatSLAM System Using Vision/Odometer}

\subsection{RatSLAM.}

Michael John Milford proposed the RatSLAM algorithm, which is a biologically inspired approach to solve SLAM problems. He presents a computational model of the rodent hippocampus. RatSLAM system contains visual odometry, an experience map, loop closure detection and feedback adjustment through sequences of familiar visual scenes.

The idea of RatSLAM comes from the biological theory of the rodent hippocampus. The scientists design the experiment focusing on the rodent in a controlled visually cued environment, which led to the discovery of cells with strikingly clear correlates to mapping tasks such as place 
cells, head direction cells, and grid cells [2, 3, 4]. Those cells were found that they contain strong spatial characteristics which are driven by recordings of neural activity for navigation and positioning. For example, place cells fire maximally when the rodent is located at a specific location in the environment, and fire to a lesser degree as the rodent moves away from this location. Head direction cells fire when the rodent's head is at specific global orientations, but their firing is not correlated to the location of the animal's body.

The RatSLAM algorithm draws upon the current understanding of spatial encoding in rat brains to perform learning and recall of maps $[5,6,7]$. The structure of the RatSLAM system is shown in Fig.1.

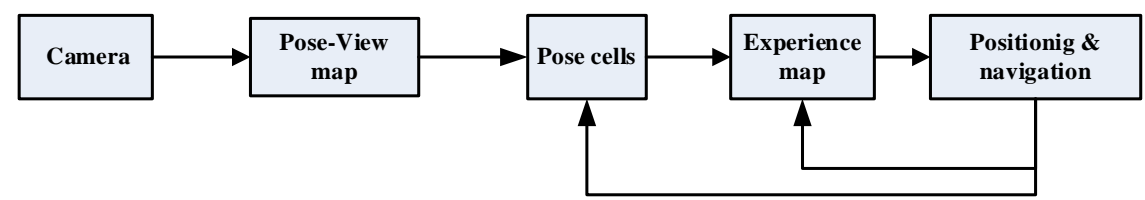

Fig.1: Structure of the RatSLAM system

The pose cells are the core of the RATSLAM system. It represents the three degree of freedom pose. Activity in the pose cells is updated by self-motion cues, and calibrated by local view. The self-motion cues are used to drive path integration in the pose cells, while the external cues trigger local view cells that are associated with pose cells through associative learning. The role of the experience map is to organize the flow of information from the pose cells, the local view cells, and the self-motion cues into a set of related spatial experiences [7, 8].

The algorithm of RATSLAM is robust and the system is fast enough to run online on a standard desktop computer or laptop while mapping a large outdoor environment. Further, it also has the ability to consistently close large loops with significant path integration errors, and to generate a coherent map by consolidating odometric information with map connectivity requirements.

\subsection{Vision/Odometer Integration based on RatSLAM.}

This paper adds odometer data into RatSLAM system in order to improve the reliability and practicability for land vehicle applications. The average value of sum of velocities by camera and odometer is provided for RatSALM system. The structure of vision/odometer integration based on RatSLAM system is shown in Fig.2.

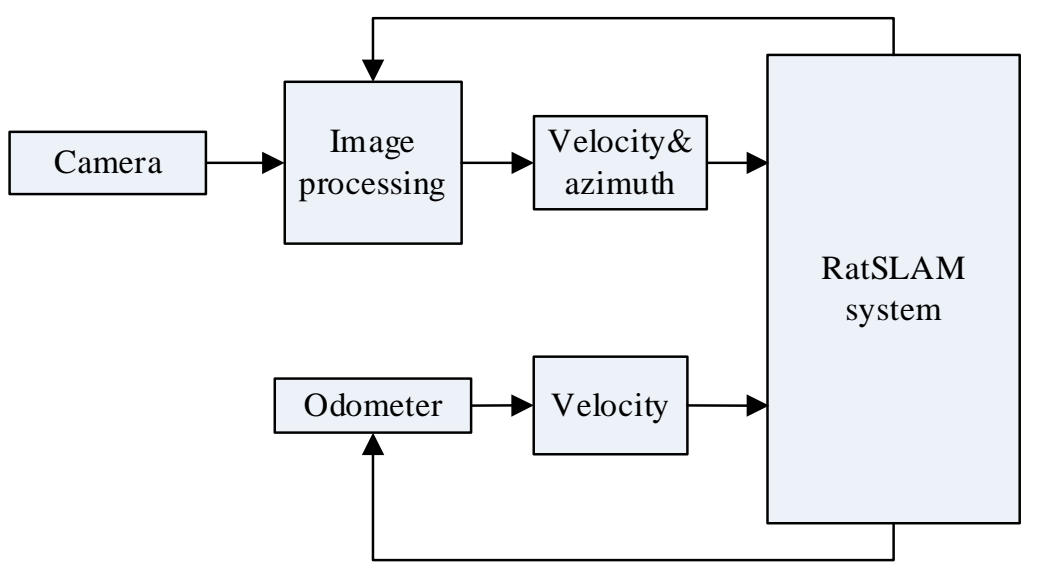

Fig.2: Structure of vision/odometer integration based on RatSLAM

\section{Experimental Results}

\subsection{Campus dataset and sensors for experiments.}

The experiments were carried out along the gymnasium of NUDT. It was a close-loop trajectory. And the test vehicle moved around the gymnasium for several loops. The road route signed by red line is shown in Fig. 3. 


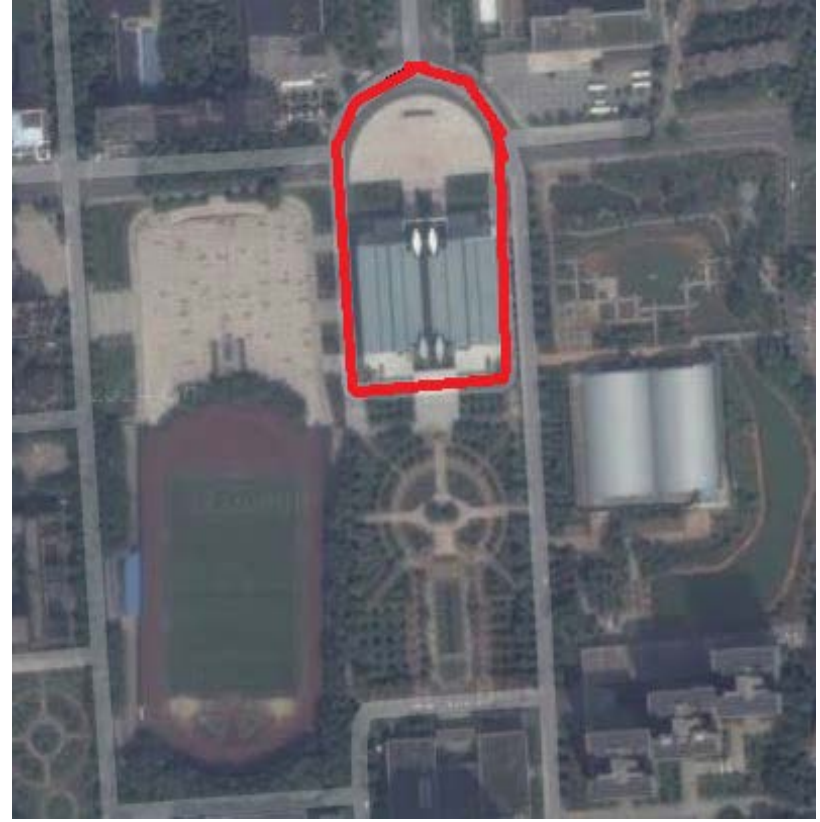

Fig.3: The campus road route

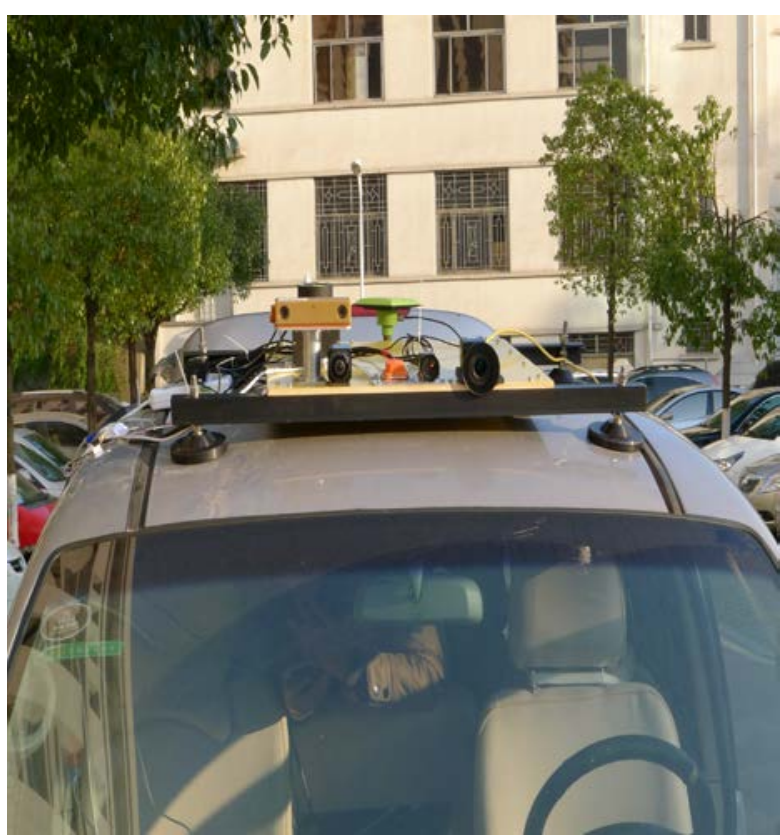

Fig.4: The camera mounted on the test vehicle

The experiment is conducted by integrating navigation grade INS system, camera, CS350A odometer and GPS navigator in the land vehicle. The ground truth of azimuth and position are offered by the navigation grade INS system and the GPS navigator. The sensors mounted on the top of the land vehicle are shown in Fig. 4.

The stereo camera shown in Fig.5 is produced by Point Grey company, named Bumblebee2 1394a. The paper only use one camera of the sensor.

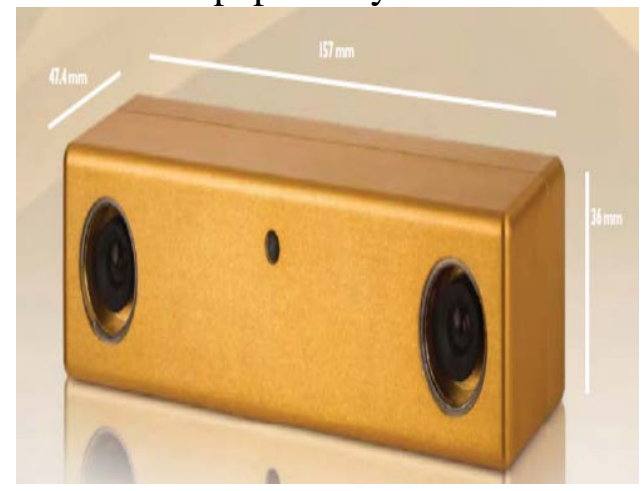

Fig.5: Bumblebee2 1394a camera

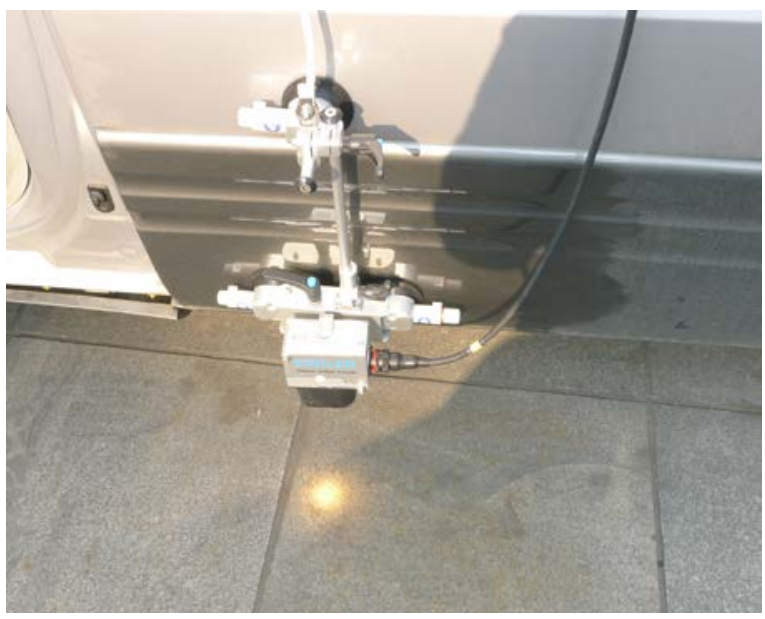

Fig.6: CS350A odometer mounted outside the test vehicle

The CS350A odometer shown in Fig.6 is produced by kistler automotive GmbH.

The navigation grade INS system was fixed in the test vehicle, which is shown in Fig. 7. 


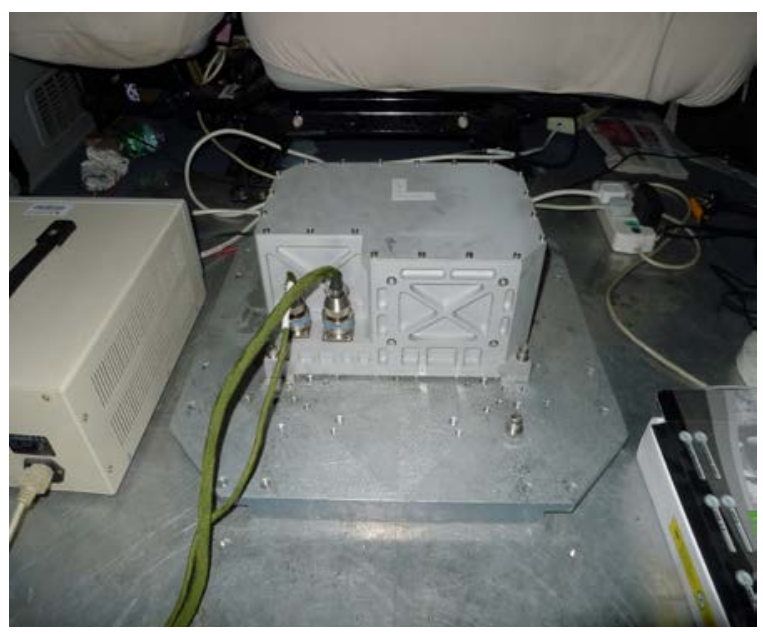

Fig.7: The navigation grade INS system

The visual image captured by the camera is illustrated in Fig.8.

\subsection{Experimental results.}

Fig.9 provides the trajectory of the vehicle estimated by vision/odometer integration algorithm based on RatSLAM. The trajectory is smooth and do not fluctuate obviously during dynamic motion. It is difficult to obtain the error curves of positioning because the accuracy of reference positioning by GPS receiver was about 5 meters. The results show that the algorithm proposed in this paper is feasible.

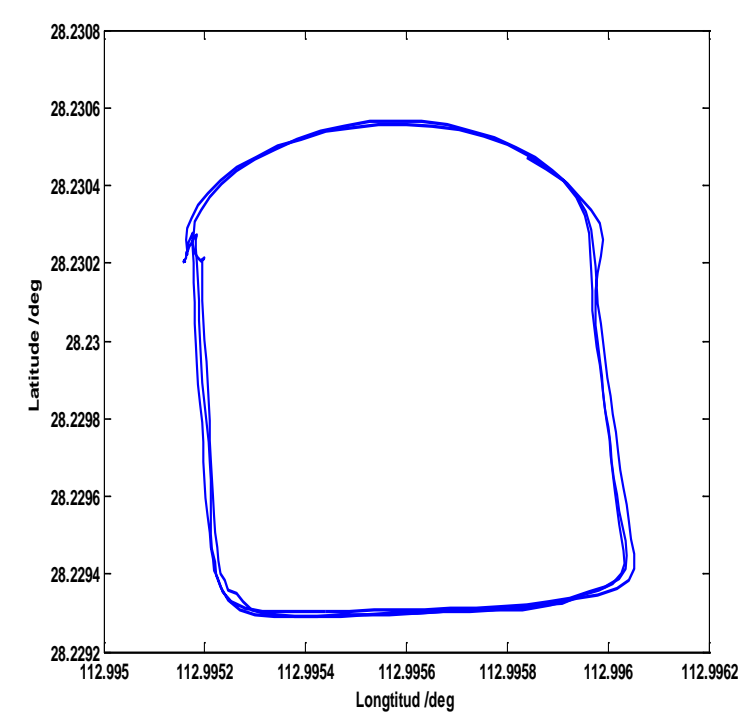

Fig.9: The estimated trajectory of the vehicle

\section{Conclusions}

Computer vision and odometer integrated autonomous navigation algorithm based on RatSLAM system for land vehicles is studied in this paper. The conclusions illustrate:

i) The structure of vision/odometer integration algorithm is presented based on RatSLAM.

ii) The algorithm studied here has been verified on real vision/odometer data collected in land vehicle tests. Campus round road experiments illustrate that the algorithm is effective and feasible.

Furthermore, more experiments should be carried out and more precision reference positioning could be built up. Further investigations are under way to study IMU(inertial measurement unit)/computer vision/odometer integrated navigation algorithms based on RatSLAM system. 
Navigation information such as position, velocity and attitude can be calculated using IMU sensors. Therefore, it should be more robust and feasible if IMU/vision/odometer integration structure is established. And RatSLAM system could be improved to be adopted by IMU/vision/odometer integration structure.

\section{Acknowledgements}

This work was financially supported by the NUDT Advanced Research Programs(JC14-03-06, JC14-03-04).

\section{References}

[1] Milford, M. J. \& Wyeth, G. F, Mapping a Suburb With a Single Camera Using a Biologically nspiredSLAM System. IEEE Trans on Robotics, 24(5), pp.1038-1053,2008.

[2] J. O'Keefe \& D. H. Conway, Hippocampal place units in the freely moving rat: Why they fire where they fire. Exp. Brain Res., 31, pp.573-590, 1978.

[3] O'Keefe, J. \& J. Dostrovsky, The hippocampus as a spatial map: preliminary evidence from unit activity in the freely moving rat, Brain Research, 34, pp. 171-175, 1971.

[4] Taube, J.S. \& R.U. Muller, Head direction cells recorded from the postsubiculum in freely moving rats. Journal of Neuroscience, 10(2), pp. 420-447, 1990.

[5] Milford, M. J., Vision-based place recognition: how low can you go?. The International Journal of Robotics Research, 32(7), pp. 766-789, 2013.

[6] Milford, M. J., Vig E., Scheirer W. \& Cox D, Vision-based Simultaneous Localization and Mapping in Changing Outdoor Environments. Journal of Field Robotics, 31(5), pp. 814-836, 2014.

[7] Milford, M. J. Robot Navigation from Nature Simultaneous Localisation, Mapping, and Path Planning Based on Hippocampal Models. Springer-Verlag Berlin Heidelberg, 2008.

[8] Wehner R. \& Srinivasan M.V. Path integration in insects. Oxford: Oxford University Press, 2003.

[9] Uğur M. Erdem, Michael J. Milford Michael \& E. Hasselmo, A hierarchical model of goal directed navigation selects trajectories in a visual environment. Neurobiology of Learning and Memory, 117(1), pp. 109-121, 2015. 